\title{
ON DISTORTION OF A CLASS OF ANALYTIC FUNCTIONS UNDER A FAMILLY OF OPERATORS
}

\author{
YOSAKU KOMATU \\ Kanazawa Institute of Technology, Kanazawa, Japan
}

\begin{abstract}
Consider a family of integral operators and a related family of differential operators, both defined on a class of analytic functions holomorphic in the unit disk, distortion properties of the real part are derived from a general aspect.
\end{abstract}

1. Integral and differential operators. Let $\widetilde{\mathcal{F}}$ denote the class of analytic functions which are holomorphic in the unit disk $E=\{|z|<1\}$. Let $\widetilde{\mathcal{F}} \operatorname{nad} \mathcal{G}$ be its subclasses consisting of $f \in \widetilde{\mathcal{F}}$ normalized by $f(0)=f^{\prime}(0)-1=0$ and $f(0)=1$, respectively. In previous papers $[3,6]$ we have observed an integral operator $\mathcal{L}(a)$ defined on $\mathcal{F}$, which is represented by

$$
\mathcal{L}(a) f(z)=a \int_{I} t^{a-2} f(z t) d t
$$

where $a$ is a positive parameter and $I$ denotes the unit interval $[0,1]$. On the other hand, Miller [7] and subsequently Altintas [1] discussed a differential operator defined by

$$
\Lambda(\alpha)=1+\alpha \frac{d}{d \log z}
$$

where $\alpha$ is a positive parameter.

As indicated in [6], the defining representation of $\mathcal{L}(a)$ shows that the normalization $f^{\prime}(0)=1$ is inessential and further it is applicable to any function of $\widetilde{\mathcal{F}}$ provided $a>1$. The interrelation between $\Lambda$ and $\mathcal{L}$ is given by the following theorem.

THEOREM 1. The differential operator $\Lambda(\alpha)$ is the inverse of the operator

$$
(\alpha+1)^{-1} \mathcal{L}((\alpha+1) / \alpha)
$$

namely

$$
\frac{1}{\alpha+1} \mathcal{L}\left(\frac{\alpha+1}{\alpha}\right) \Lambda(\alpha)=\mathrm{id},
$$

or, in other words, $\left(a(a-1)^{-1}\right) \Lambda\left((a-1)^{-1}\right)$ is the inverse of $\mathcal{L}(a)$.

1991 Mathematics Subject Classification: Primary 46C20; Secondary 32G81.

The paper is in final form and no version of it will be published elsewhere. 
P r o of. Direct calculation yields

$$
\begin{aligned}
& \Lambda(\alpha) \frac{1}{\alpha+1} \mathcal{L}\left(\frac{\alpha+1}{\alpha}\right) f(z)=\Lambda(\alpha) \frac{1}{\alpha} \int_{I} t^{\frac{1}{\alpha-1}} f(z t) d t=\frac{1}{\alpha} \int_{I} t^{\frac{1}{\alpha-1}}\left(f(z t)+\alpha z t f^{\prime}(z t)\right) d t \\
& =\int_{I} \frac{\partial}{\partial t}\left(t^{\frac{1}{\alpha}} f(z t)\right) d t=f(z) .
\end{aligned}
$$

The iterated sequence $\left\{\mathcal{L}(a)^{n}\right\}_{n=1}^{\infty}$ arises automatically. Moreover, as shown in [3], it can be interpolated into a family $\left\{\mathcal{L}(a)^{\lambda}\right\}_{\lambda>0}$ depending on a continuous parameter $\lambda$, in such a manner that it possesses the structure of semigroup; namely, it satisfies $\mathcal{L}(a)^{\lambda} \mathcal{L}(a)^{\mu}=\mathcal{L}(a)^{\lambda+\mu}$. Further it's every member is explicity represented by

$$
\mathcal{L}(a)^{\lambda} f(z)=\frac{a^{\lambda}}{\Gamma(\lambda)} \int_{I} t^{a-2}\left(\log \frac{1}{t}\right)^{\lambda-1} f(z t) d t,
$$

$\mathcal{L}(a)^{0}$ being understood to be the identity. While this representation has been derived for $f \in \mathcal{F}$ and $a>0$, it applies also to any $f \in \mathcal{G}$ provided $a>1$.

According to this extension, Theorem 1 leads to the following corollary which may be regarded as a relation defining the fractional integral operator $\Lambda(\alpha)^{-\lambda}$.

Corollary 1. If $\alpha>0$ and $\lambda \geq 0$, we have

$$
\frac{1}{(\alpha+1)^{\lambda}} \mathcal{L}\left(\frac{\alpha+1}{\alpha}\right)^{\lambda} \Lambda(\alpha)^{\lambda}=\mathrm{id} .
$$

For the later purpose we notice the analytic prolongation of $\mathcal{L}(a)^{\lambda}$. In fact, as shown in [5], this operator is analytically prolongable with respect to $\lambda$ and $a$ within singlevaluedness into the whole complex plane $(\lambda, a)$ cut along the negative real axis on the $a$-plane. While $\mathcal{L}(a)^{\lambda}$ behaves on the negative real axis of the $a$-plane also analytically, provided $a \neq-1,-2, \ldots$ if $\operatorname{Re} \lambda>0, a \neq 0$ if $\operatorname{Re} \lambda<0$, and $a \neq 0,-1,-2, \ldots$ if $\operatorname{Re}$ $\lambda=0$; however, the operator shows after prolongation many-valuedness when $a$ crosses the negative real axis, unless $\lambda$ coincides with an integer.

Accordingly, in view of Corollary 1 , the operator $\Lambda(\alpha)^{\lambda}$ also possesses a similar prolongability with respect to the pair of parameters $\lambda$ and $a$.

2. Distorsion of the real part. In the following lines we restrict ourselves to the case of $\lambda>0$ and $\alpha>0$. It has been shown by Miller [7] and Altintas [1] that if $f \in \mathcal{G}$ satisfies $\operatorname{Re} \Lambda(\alpha) f(z)>\beta$, then $\operatorname{Re} f(z)>\beta$.

While this result has been partly improved in [5], it can be further generalized as in the following form.

THEOREM 2. If $f \in \widetilde{\mathcal{F}}$ with $f(0)=a_{0}+i b_{0}$ and $a_{0}>\beta_{*}$ satisfies in $E$ the inequality $\operatorname{Re} \Lambda(\alpha)^{\lambda} f(z)>\beta_{*^{\prime}}$, then

$$
\operatorname{Re} f(z)>\beta_{*}+\left(a_{0}-\beta_{*}\right) \Psi(\lambda, \alpha)
$$

where

$$
\Psi(\lambda, \alpha)=\frac{1}{\alpha^{\lambda} \Gamma(\lambda)} \int_{I} t^{\frac{1}{\alpha-1}}\left(\log \frac{1}{t}\right)^{\lambda-1} \frac{1-t}{1+t} d t
$$


The function $\widehat{f}$ of the form

$$
\widehat{f}(z ; \beta)=\beta+\frac{\widehat{a}_{0}-\beta}{\alpha^{\lambda} \Gamma(\lambda)} \int_{I} t^{\frac{1}{\alpha-1}}\left(\log \frac{1}{t}\right)^{\lambda-1} \frac{1-\varepsilon z t}{1+\varepsilon z t} d t+i \widehat{b}_{0}
$$

with $\widehat{f}(0, \beta)=\widehat{a}_{0}+i b_{0}$ and $|\varepsilon|=1$ is extremal in the following sense:

$$
\inf _{z \in E} \operatorname{Re} \Lambda(\alpha)^{\lambda} \widehat{f}\left(z ; \beta_{*}\right)=\beta_{*}
$$

and

$$
\inf _{z \in E} \operatorname{Re} \widehat{f}\left(0, \beta_{*}\right)=\beta_{*}+\left(a_{0}-\beta_{*}\right) \Psi(\lambda, \alpha) .
$$

Proof. We first note that

$$
\mathcal{L}(a)^{\lambda} 1=\frac{a^{\lambda}}{\Gamma(\lambda)} \int_{I} t^{a-2}\left(\log \frac{1}{t}\right)^{\lambda-1} d t=\left(\frac{a}{a-1}\right)^{\lambda},
$$

whence follows, in view of analytic prolongability, for any constant $c$,

$$
\Lambda(a)^{\lambda} c=\frac{1}{(\alpha+1)^{\lambda}} \mathcal{L}\left(\frac{\alpha+1}{\alpha}\right)^{\lambda} c=\frac{1}{(\alpha+1)^{\lambda}}(\alpha+1)^{\lambda} c=c .
$$

Consequently, Harnack inequality yields

$$
\operatorname{Re} \frac{\Lambda(\alpha)^{\lambda} f(\zeta)-\beta_{*}}{a_{0}-\beta_{*}} \geq \frac{1-|\zeta|}{1+|\zeta|}
$$

for $\zeta \in E$, whence follows

$$
\operatorname{Re} \Lambda(\alpha)^{\lambda} f(z t)>\beta_{*}+\left(a_{0}-\beta_{*}\right) \frac{1-t}{1+t}
$$

for $z \in E$ and $t \in(0,1]$. Consequently, basing on the relation

we obtain

$$
\begin{aligned}
f(z) & =\Lambda(\alpha)^{-\lambda} \Lambda(\alpha)^{\lambda} f(z) \\
& =\frac{1}{(\alpha+1)^{\lambda}} \mathcal{L}\left(\frac{\alpha+1}{\alpha}\right)^{\lambda} \Lambda(\alpha)^{\lambda} f(z) \\
& =\frac{1}{\alpha^{\lambda} \Gamma(\lambda)} \int_{I} t^{\frac{1}{\alpha-1}}\left(\log \frac{1}{t}\right)^{\lambda-1} \Lambda(\alpha)^{\lambda} f(z t) d t,
\end{aligned}
$$

$$
\begin{aligned}
\operatorname{Re} f(z) & >\frac{1}{\alpha^{\lambda} \Gamma(\lambda)} \int_{I} t^{\frac{1}{\alpha-1}}\left(\log \frac{1}{t}\right)^{\lambda-1}\left(\beta_{*}+\left(a_{0}-\beta_{*}\right)\right) \frac{1-t}{1+t} d t \\
& =\beta_{*}+\left(a_{0}-\beta_{*}\right) \Psi(\lambda, \alpha) .
\end{aligned}
$$

Next the function $\widehat{f}$ given in theorem may be written in the form

$$
\widehat{f}(z ; \beta)=\beta+\left(\widehat{a}_{0}-\beta\right) \Lambda(\alpha)^{-\lambda} \frac{1-\varepsilon z}{1+\varepsilon z}+i \widehat{b}_{0} .
$$

whence follows

$$
\Lambda(\alpha)^{\lambda} \widehat{f}(z ; \beta)=\beta+\left(\widehat{a}_{0}-\beta\right) \frac{1-\varepsilon z}{1+\varepsilon z}+i \widehat{b}_{0} .
$$

Thus the last assertion follows readily.

Corollary 2. If $f \in \widetilde{\mathcal{F}}$ with $f(0)=a_{0}+i b_{0}$ and $a_{0}<\beta^{*}$ satisfies in $E$ the inequality $\operatorname{Re} \Lambda(\alpha)^{\lambda} f(z)<\beta^{*}$, then

$$
\operatorname{Re} f(z)<\beta^{*}-\left(\beta^{*}-a_{0}\right) \Psi(\lambda, \alpha),
$$


where $\Psi$ is the expression defined in Theorem 2. The function $\widehat{f}$ given in Theorem 2 is extremal in the sense:

$$
\begin{gathered}
\sup _{z \in E} \operatorname{Re} \Lambda(\alpha)^{\lambda} \widehat{f}\left(z ; \beta^{*}\right)=\beta^{*}, \\
\sup _{z \in E} \operatorname{Re} \widehat{f}\left(z ; \beta^{*}\right)=\beta^{*}-\left(\beta^{*}-\widehat{a}_{0}\right) \Psi(\lambda, \alpha) .
\end{gathered}
$$

Proof. We have only to apply Theorem 2 to the function $-f$ instead of $f$ and to replace the quantities $\beta_{*}$ and $a_{0}$ by $-\beta^{*}$ and $-a_{0}$, respectively.

Now, in view of the interrelation between $\Lambda(\alpha)$ and $\mathcal{L}(a), a=(\lambda+1) / \lambda$, stated in Corollary 1 and the analytic prolongability with respect to $\lambda$, the semigroup character of $\left\{\mathcal{L}(a)^{\lambda}\right\}_{\lambda}$ implies that of $\left\{\Lambda(a)^{\lambda}\right\}_{\lambda}$; namely, $\Lambda(\alpha)^{\lambda}=\Lambda(\alpha)^{\mu} \Lambda(\alpha)^{\lambda-\mu}$ for $0<\mu<\lambda$. Hence, by repeated application of Theorem 2, we see that $\Lambda(\alpha)^{\lambda} f(z)>\beta_{*}$ implies

$$
\begin{gathered}
\operatorname{Re} \Lambda(\alpha)^{\mu} f(z)>\beta_{*}+\left(a_{0}-\beta_{*}\right) \Psi(\lambda-\lambda, \alpha), \\
\operatorname{Re} f(z)>\beta_{*}+\left(a_{0}-\beta_{*}\right) \Psi(\lambda-\mu, \alpha)+\left(a_{0}-\left(\beta_{*}+\left(a_{0}-\beta_{*}\right) \Psi(\lambda-\mu, \alpha)\right) \Psi(\mu, \alpha)\right. \\
=\beta_{*}+\left(a_{0}-\beta_{*}\right) \Psi(\lambda-\mu, \alpha)+(1-\Psi(\lambda-\mu, \alpha)) \Psi(\mu, \alpha) .
\end{gathered}
$$

by comparing the lower bound $B_{*}^{(1)}=\beta_{*}+\left(a_{0}-\beta_{*}\right) \Psi(\lambda, \alpha)$ for $\operatorname{Re} f(z)$ given in Theorem 2 with the bound $B_{*}^{(2)}$ obtained in the last expression, their difference becomes

$$
B_{*}^{(1)}-B_{*}^{(2)}=\left(a_{0}-\beta_{*}\right)((1-\Psi(\mu, \alpha))(1-\Psi(\lambda-\mu, \alpha)-(1-\Psi(\lambda, \alpha))) .
$$

It may be directly shown that the logarithm of the quantity

$$
1-\Psi(\lambda, \alpha)=\frac{1}{\alpha^{\lambda} \Gamma(\lambda)} \int_{I} t^{\frac{1}{\alpha-1}}\left(\log \frac{1}{t}\right)^{\lambda-1} \frac{2 t}{1+t} d t
$$

possesses the subadditivity with respect to $\lambda$, i.e.,

$$
1-\Psi(\lambda, \alpha)<(1-\Psi(\mu, \alpha))(1-\Psi(\lambda-\mu, \alpha)),
$$

$0<\mu<\lambda$, whence follows $B_{*}^{(1)}>B_{*}^{(2)}$ verifying that the estimate based on Theorem 2 at one stretch is better than the estimate obtained by repeated steps. Concerning the upper bound given in Corollary 2, the circumstance is quite similar.

3. Remarks to the bounds of distorsion. The quantity $\Psi(\lambda, \mu)$ is nothing but the quantity $\Phi(\lambda, 1 / \alpha)$ dealt with in [3]. It can be expressed in the series from

$$
\left.\Psi(\lambda, \alpha)=1+\frac{1}{\alpha^{\lambda}} \sum_{\nu=2}^{\infty} \frac{(-1)^{\nu}}{\left(\nu+\alpha^{-1}-1\right.}\right)^{\lambda}
$$

and if, in particular. $\alpha^{-1}=k$ is a positive integer, $\Psi$ is alternatively expressible in the form

$$
\Psi\left(\lambda, \frac{1}{k}\right)=1+2(-1)^{k-1} k^{\lambda}\left(\left(1-2^{1-\lambda}\right) \zeta(\lambda)+\sum_{\kappa=1}^{k} \frac{(-1)^{\kappa}}{\kappa^{\lambda}}\right)
$$

$\zeta$ denoting Riemann zeta function and the value of $\left(1-2^{1-\lambda}\right) \zeta(\lambda)$ at $\lambda=1$ being understood to be its limit value $\log 2$.

It has been also noted $\Psi(\lambda, \alpha)$ possesses monotonicity with respect to each argument. In fact, for any fixed $\alpha>0$ or fixed $\lambda>0, \Psi(\lambda, \alpha)$ increases strictly from 0 to 1 when $\lambda$ or $\alpha$ increases from 0 to $\infty$, respectively. 
Now, we deal with the sequence $\{\Psi(\lambda, 1 / k)\}_{k=1}^{\infty}$ for any fixed $\lambda$. In view of the abovestated expression for $\Psi$, it follows readily that it satisfies the recurrence relation

$$
\Psi\left(\lambda, \frac{1}{k+1}\right)=\left(1+\frac{1}{k+1}\right)^{\lambda}-1-\left(1+\frac{1}{k}\right)^{\lambda} \Psi\left(\lambda, \frac{1}{k}\right) .
$$

For $\lambda=1$, we have

$$
\Psi(1,1)=2 \log 2-1,
$$

while for even integer $\lambda=2 m,(m=1,2, \ldots)$, we have

$$
\Psi(2 m, 1)=2\left(1-2^{1-2 m}\right) \zeta(2 m)-1=\left(1-2^{1-2 m}\right)(2 \pi)^{2 m} \frac{(-1)^{m-1} B_{2 m}}{(2 m) !}-1,
$$

where $B$ 's denote Bernoulli numbers defined by the generating function

$$
\frac{t}{\exp t-1}=\sum_{n=1}^{\infty} \frac{B_{n}}{n !} t^{n}
$$

In particular, for lower values of $m$, the numerical valuse off $B$ 's are given by

$$
\begin{gathered}
B_{2}=\frac{1}{6}, \quad B_{4}=-\frac{1}{30}, \quad B_{6}=\frac{1}{42}, \quad B_{8}=\frac{1}{10}, \\
B_{10}=\frac{5}{66}, \quad B_{12}=-\frac{691}{2730}, \quad B_{14}=\frac{7}{6}, \ldots . .
\end{gathered}
$$

Hence, in view of the above-mentioned recurrence relation, the numerical values of $\Psi(\lambda, 1 / k)$ with $\lambda=1$ or $2 m, m=1,2, \ldots$ and $k=1,2, \ldots$ will be successively determined. For instance,

$$
\begin{aligned}
& \Psi(1,1)=2 \log 2-1=0,3862 \ldots, \\
& \Psi\left(1, \frac{1}{2}\right)=3-4 \log 2=0,2274 \ldots, \\
& \Psi\left(1, \frac{1}{3}\right)=6 \log 2-4=0,1588 \ldots ; \\
& \Psi(2,1)=\frac{\pi^{2}}{6}-1=0,6449 \ldots, \\
& \Psi\left(2, \frac{1}{2}\right)=7-\frac{2 \pi^{2}}{3}=0,4202 \ldots, \\
& \Psi\left(2, \frac{1}{3}\right)=\frac{3 \pi^{2}}{2}-\frac{29}{2}=0,3044 \ldots ; \\
& \Psi(4,1)=\frac{7 \pi^{4}}{360}-1=0,8940 \ldots, \\
& \Psi\left(4, \frac{1}{2}\right)=31-\frac{14 \pi^{4}}{45}=0,6949 \ldots, \\
& \Psi\left(4, \frac{1}{3}\right)=\frac{567 \pi^{4}}{360}-\frac{1223}{8}=0,5443 \ldots .
\end{aligned}
$$

4. Generalization of the distorsion. The background of the proof of Theorem 2 is based on a simple idea of subordination.

Let $D$ be any simply-connected domain laid the $w$-plane which is the image of $E$ by a mapping $w=W(z)$ satisfyin $W(0)=f(0)$. Te Green function of the domain $D$ with 
pole at $W(0)$ is given by $\log \left|W^{-1}(w)\right|^{-1}$ and hence its level curve $C_{t}$ of height $\log t^{-1}$ is expressed by $\left\{\left|W^{-1}(w)\right|=t\right\}$. The preimage of $C_{t}$ is the concentric circumference $\{|z|=t\}$. The image of $\{|z|<t\}$ is the interior of $C_{t}$ which will be denoted by $D_{t}$.

If $\Lambda(\alpha)^{\lambda} f(z) \subset D$, then it is subordinate to $W(z)$ and hence $\Lambda(\alpha)^{\lambda} f(z t) \subset D$ for any $z \in E$ and $t \in I$. Consequently, for any $z \in E$, the quantity

$$
f(z)=\Lambda(\alpha)^{-\lambda} \Lambda(\alpha)^{\lambda} f(z t)=\frac{1}{\alpha^{\lambda} \Gamma(\lambda)} \int_{I} t^{\frac{1}{\alpha-1}}\left(\log \frac{1}{t}\right)^{\lambda-1} \Lambda(\alpha)^{\lambda} f(z t) d t
$$

may be regarded as a weighted mean of $\Lambda(\alpha)^{\lambda} f(z t) \in D_{t}$ along $I$ with a positive weight.

Thus, we can state a theorem generalizing Theorem 2 and its Corollary 2:

THEOREM 3. Let $f \in \widetilde{\mathcal{F}}$. Let $D$ be a simply-connected domain and $D$ the interior of the level curve of height $\log t^{-1}$ of its Green function with pole at $f(0)$. Then $\Lambda(\alpha)^{\lambda} f(E) \subset D$ with $\alpha>0$ and $\lambda>0$ implies

$$
\Lambda(\alpha)^{\lambda} f(\{|z|<t\}) \subset D_{t}
$$

In particular, if $D$ is symmetric with respect to the horizontal line $\{\operatorname{Im} w=\operatorname{Im} f(0)\}$, then $\operatorname{Re} \Lambda(\alpha)^{\lambda} f(E) \subset D$ implies

$$
\Omega_{*}<n \operatorname{Re} f(z)<\Omega^{*}
$$

where $\Omega_{*}$ and $\Omega^{*}$ are given by

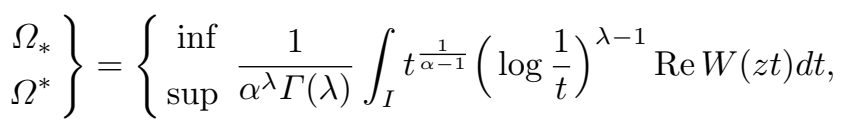

$W$ being a function univalently mapping $E$ onto $D$ which satisfies $W(0)=f(0)$; here inf and sup are concerned in $z$ lying on the circumference $\{|z|=1\}$.

Proof. The first part of the assertion follows readily from the subordination principle. For the second particular case, we have only to notice that the symmetry of $D$ with respect to the line $\{\operatorname{Im} w=\operatorname{Im} f(0)\}$ implies that of $D_{t}$ for any $t \in I$.

As an example of Theorem 3, we state here a result obtained by applying it to the case where $D$ is a parallel strip. It relates to a result in [2].

Corollary 3. If, in Theorem 3, D is a parallel strip $\left\{\beta_{*}<\operatorname{Re} w<\beta^{*}\right\}$, then the bounds of $\operatorname{Re} f(z)$ are given by

$$
\left.\begin{array}{l}
\Omega_{*} \\
\Omega^{*}
\end{array}\right\}=\frac{1}{\alpha^{\lambda} \Gamma(\lambda)} \int_{I} t^{\frac{1}{\alpha-1}}\left(\log \frac{1}{t}\right)^{\lambda-1} W(\mp t) d t,
$$

denoting the function mapping $E$ onto $D$ with $W(0)=a_{0}+i b_{0}$ :

where

$$
W(0)=\frac{2\left(\beta^{*}-\beta_{*}\right)}{\pi} \arctan \frac{z+\tau}{1+\tau z}+\frac{\beta^{*}+\beta_{*}}{2}+i b_{0}
$$

$$
\tau=-\tan \left(\frac{\pi}{4} \frac{\beta^{*}+\beta_{*}-2 a_{0}}{\beta^{*}-\beta_{*}}\right) .
$$

The extremal function $\widehat{f}$ is of the form

$$
\widehat{f}(z)=\Lambda(\alpha)^{\lambda} W(z)
$$


with $a_{0}=\widehat{a}_{0}, b_{0}=\widehat{b}_{0}$ and the respective lower and upper bounds are attained at boundary points $z=-1$ and $z=+1$, respectively, where $\widehat{f}$ behaves holomorphically.

Proof. Since the image strip $\left\{\beta_{*}<\operatorname{Re} w<\beta^{*}\right\}$ of $E$ by the mapping $w=W(z)$ is convex, the image $W(\{|z|<t\})$ is so also for any $t \in I$. Consequently, in view of the symmetry with respect to the line $\left\{\operatorname{Im} w=b_{0}\right\}$, the minimum and maximum of $\operatorname{Re} W(z)$ as well as off $\operatorname{Re} \Lambda(\alpha)^{\lambda} W(z)$ on the circumference $\left.\{|z|=t\}\right)$ are attained at $z=-t$ and $z=+t$, respectively. Hence, in view of Theorem 3, the assertion follows.

The corollary just stated may be regarded as an improvement of Theorem 2 together with its Corollary 2. As noticed in [2], Corollary 3 reduces to Theorem 2 and Corollary 2 as the limit casses of $\beta^{*} \rightarrow+\infty$ and $\beta_{*} \rightarrow-\infty$, respectively. It is shown that if we retain $\beta_{*}$ and observe the limit case of $\beta^{*} \rightarrow+\infty$, the lower bound for $\operatorname{Re} f(z)$ given in Corollary 3 reduces to that given in Theorem 2 . In fact, it is verified that as $\beta^{*} \rightarrow+\infty$, we obtain

$$
\begin{gathered}
\tau=-1+\frac{\pi\left(a_{0}-\beta_{*}\right)}{\beta^{*}}+o\left(\frac{1}{\beta^{*}}\right), \\
\arctan \frac{\tau-t}{1-\tau t}=-\frac{\pi}{4}+\frac{\pi\left(a_{0}-\beta_{*}\right)}{2 \beta^{*}} \frac{1-t}{1+t}+o\left(\frac{1}{\beta^{*}}\right),
\end{gathered}
$$

every $o$-notation being uniform, and hence

$$
\begin{aligned}
\Omega_{*} & =\frac{2\left(\beta^{*}-\beta_{*}\right)}{\pi} \frac{1}{\alpha^{\lambda} \Gamma(\lambda)} \int_{I} t^{\frac{1}{\alpha-1}}\left(\log \frac{1}{t}\right)^{\lambda-1} \arctan \frac{\tau-t}{1-\tau t} d t+\frac{\beta^{*}+\beta_{*}}{2} \\
& =\beta_{*}+\left(a_{0}-\beta_{*}\right) \Psi(\lambda, \alpha)+o(1) .
\end{aligned}
$$

Similarly, as $\beta_{*} \rightarrow-\infty$, we obtain

$$
\Omega^{*}=\beta^{*}-\left(\beta^{*}-a_{0}\right) \Psi(\lambda, \alpha)+o(1) .
$$

\section{References}

[1] O. Atlantas, On the coefficients of function majorized by univalent functions, Hacetteppe Bull. Nat. Sci. - Engrg. 10 (1981), 23-30.

[2] Y. Komatu, On distorsion of the real part in a class of analy functions related to fractional integration. Compl. Var. 7 (1986), 97-106.

[3] - On a family of integral operators related to fractional calculus, Kodai Math. J. 10 (1987), 20-38.

[4] -, On analytic differential operator, Unival. Func., Fract. Calc., Appl. Ellis Horwood Ltd., UK (1989), 103-112.

[5] -, On analytic prolongation of a family of operators, Mathematica, Cluj-Napoca 32 (55) (1990), 141-145.

[6] -, On distorsion properties of analytic operators, Kodai Math. J. 15 (1992), 1-10.

[7] S. Miller, Differential inequalities and Caratheodory functions, Bull. Amer. Math. Soc. 81 (1975), 79-81. 